\title{
Covariant Prolongation Structure, Conservation Laws and Soliton Solutions of the Gross-Pitaevskii Equation in the Bose-Einstein Condensate
}

\author{
Souleymanou Abbagari, 1,2,3,4, Hamadou Halidou'2,3,4, Thomas B. Bouetou ${ }^{3,4}$, Timoleon C. Kofane ${ }^{2,4}$ \\ ${ }^{1}$ Department of Basic Science, Law and Humanities, Institute of Mines and Petroleum Industries, University of Maroua, Maroua, \\ Cameroon \\ ${ }^{2}$ Laboratory of Mechanics, Materials and Structures, Department of physics, University of Yaounde I, Yaounde, Cameroon \\ ${ }^{3}$ National Advanced School of Engineering, University of Yaounde I, Yaounde, Cameroon \\ ${ }^{4}$ Centre d'Excellence en Technologies de l'Information et de la Communication (CETIC), University of Yaounde I, Yaounde, Cameroon \\ Email: abbagaris@yahoo.fr
}

How to cite this paper: Abbagari, S., Halidou, H., Bouetou, T.B. and Kofane, T.C. (2017) Covariant Prolongation Structure, Conservation Laws and Soliton Solutions of the Gross-Pitaevskii Equation in the BoseEinstein Condensate. Journal of Applied Mathematics and Physics, 5, 1411-1423. https://doi.org/10.4236/jamp.2017.57116

Received: May 30, 2017

Accepted: July 15, 2017

Published: July 18, 2017

Copyright $\odot 2017$ by authors and Scientific Research Publishing Inc. This work is licensed under the Creative Commons Attribution International License (CC BY 4.0).

http://creativecommons.org/licenses/by/4.0/

(c) † Open Access

\begin{abstract}
In this paper, we investigate the Gross-Pitaevskii (GP) equation which describes the propagation of an electron plasma wave packet with a large wavelength and small amplitude in a medium with a parabolic density and constant interactional damping by the Covariant Prolongation Structure Theory. As a result, we obtain general forms of Lax-Pair representations. In addition, some hidden structural symmetries that govern the dynamics of the GP equation such as $\mathrm{SL}(2, \mathrm{R}), \mathrm{SL}(2, \mathrm{C})$, Virasoro algebra, $\mathrm{SU}(1,1)$ and $\mathrm{SU}(2)$ are unearthed. Using the Riccati form of the linear eigenvalue problem, infinite number of conservation laws of the GP equation is explicitly constructed and the exact analytical soliton solutions are obtained by employing the simple and straightforward Hirota's bilinear method.
\end{abstract}

\section{Keywords}

Gross-Pitaevskii Equation, Covariant Prolongation Structure Theory, Hidden Structural Symmetries, Hirota's Bilinear Method

\section{Introduction}

Nonlinear evolution equations (NLEEs) have been studied in diverse areas in physics and applied mathematics such as plasma physics, nonlinear optical fibers, condensed matter etc [1] [2] [3]. The inhomogeneous nonlinear Schrödinger equation among those (NLEEs) describes the propagation of an electron plasma 
wave packet with a large wavelength and small amplitude in a medium with a parabolic density and constant interactional damping [4] [5].

As it is known, the investigation of integrability of certain kinds of (NLEEs) by many researchers has generated a great deals of attention over the past years and now many methods to analyze the complete integrability of nonlinear evolution equations are developed. Among them, Wahlquist and Estabrook's prolongation technique [6]-[23] is a powerful and systematic method to test the integrability of the physically important nonlinear evolution equations. By virtue of these techniques, Morris [23] has analyzed the multi-component nonlinear Schrödinger equations.

In 1980's, based upon the nonlinear connection theory proposed by Lu et al. [24] Guo et al. [25] [26] [27] have proposed a covariant geometry theory for the prolongation structure of the nonlinear evolution equation.

Since the experimental realization of the Bose-Einstein condensate (BEC) for rubidium and sodium [28] [29], study on the properties of the BEC systems has attracted peoples attention [30] [31]. The BECs have also been observed in certain atomic gases such as the lithium, hydrogen, helium and potassium atomic gases [32] [33]. For a cigar-shaped BEC at a relatively low density, when the energy of the two-body interaction is much less than the kinetic energy in the transverse direction, the BEC system can be treated as quasi-one-dimensional [34] [35]. In the following paper, we plan to investigate the following Gross-Pitaevskii equation in the Bose-Einstein condensate [36] [37] by the covariant prolongation structure theory:

$$
i q_{t}+q_{x x}+2 \mu^{2}|q|^{2} q+\left(i \beta-\alpha x+\beta^{2} x^{2}\right) q=0,
$$

where $q(x, t)$ represents the mean-field wave function of the Bose-Einstein condensate; $x$ and $t$ are the normalized distance and retarded time, respectively; $\alpha$ and $\beta$ are all the real numbers; $\mu$ is the nonlinearity parameter; $i \beta$ is the gain $(\beta<0)$ or loss $(\beta>0)$ term; $-\alpha x$ represents the linear external potential, while $\beta^{2} x^{2}$ accounts for the harmonic external potential.

In soliton theory and other fields of science and engineering, the language of technical computing played a very important role in analytically dealing with large amounts of complicated and tedious algebraic calculations [38] [39]. In this paper, we will employ symbolic computation to study the integrability aspects and relevant soliton structures of Gross-Pitaevskii equation in the Bose-Einstein condensate [36] [37].

However, to our knowledge, for Equation (1), Lax-Pair, Conservation laws, multiple soliton solutions via Hirota's method and symbolic computation have not been discussed yet. Motivated by the above, a Lax-Pair based on the generators of some hidden structural symmetries governed the dynamics of the (GP) equation will be got in section 3 . In section 4, an infinite sequence of conservation laws of Equation (1) are obtained. In addition, in section 5 we present the exact one and two soliton solutions of the Gross-Pitaevskii Equation 
in the Bose-Einstein condensate. Finally, the conclusion will be addressed in section 6 .

\section{Covariant Theory for Prolongation Structure of Nonlinear Evolution Equations}

For a given $(1+1)$-dimensional nonlinear evolution equation with two independent variables $x$ and $t$, we can introduce a set of new variables $X=\left\{x_{1}, x_{2}, x_{3}, \cdots, x_{n}\right\}=\left\{x, t, x_{3}, \cdots, x_{n}\right\}$ and define a set of 2 -forms $I=\left\{\sigma^{j}\right\}$ such that it constitutes a differential closed ideal, which lead to the $(1+1)$ -dimensional nonlinear evolution equation if the ideal is restricted on the solution space $S=\left\{x, t, x_{3}(x, t), \cdots, x_{n}(x, t)\right\}$.

Now we take $X$ as the base space, $Y=\{y\}=\left\{y^{1}, \cdots, y^{i}\right\}$ named prolongation variables as the fiber space and $G$ as the structure group generated by the prolongation algebra $g$. We can consider a principle bundle $P(X, G)$ and the bundle $E(X, Y, G, P)$ associated with $P$. Define the local cross-section on $E$, $\tau: X \rightarrow E$, and its covariant derivatives:

$$
\omega^{i}=\mathrm{d} y^{i}+\Gamma_{r}^{i}(X, y) \mathrm{d} x^{r}=\mathrm{d} y^{i}+\Gamma_{r}^{a}(X) \lambda_{a}^{i}(y) \mathrm{d} x^{r},
$$

where $i$ is the dimension of the representation space of the prolongation algebra, $\Gamma_{r}^{a}(X)$ are the coefficients of the connection on the principal bundle $P$ and $\lambda_{a}^{i}(y)$ are the coefficients of the generators of the prolongation algebra $g$.

Then we introduce the following connection 1-forms:

$$
L_{k}^{i}=L_{k r}^{i} \mathrm{~d} x^{r}=\left[\lambda_{a}^{i}(y) \frac{\partial \lambda_{k}^{a}(y)}{\partial x^{r}}+C_{c b}^{a} \Gamma_{r}^{b}(x) \lambda_{k}^{c}(y) \lambda_{a}^{i}(y)\right] \mathrm{d} x^{r},
$$

$C_{c b}^{a}$ are the structure constants of the prolongation algebra $g$. Using the induced connection $L_{k r}^{i}$, we can define the following covariant exterior derivative:

$$
D^{*} \omega^{i}=\mathrm{d} \omega^{i}+L_{j}^{i} \wedge \omega^{j}=-\frac{1}{2} F_{r s}^{a} \lambda_{a}^{i} \mathrm{~d} x^{r} \wedge \mathrm{d} x^{s}+\frac{1}{2} M_{j k}^{i} \lambda_{a}^{i} \omega^{j} \wedge \omega^{k},
$$

where $F_{r s}^{a}$ and $M_{j k}^{i}$ are the curvature coefficients on $P$ and the torsion coefficients in the fiber space $Y$, respectively, and given by

$$
\begin{gathered}
F_{r s}^{a}(X)=\frac{\partial \Gamma_{r}^{a}(X)}{\partial x^{s}}-\frac{\partial \Gamma_{s}^{a}(X)}{\partial x^{r}}+\Gamma_{r}^{b}(X) \Gamma_{s}^{c}(X) C_{c b}^{a}, \\
M_{j k}^{i}(Y)=\lambda_{j}^{a}(y) \frac{\partial \lambda_{a}^{i}(y)}{\partial y^{k}}-\lambda_{k}^{a}(y) \frac{\partial \lambda_{a}^{i}(y)}{\partial y^{j}} .
\end{gathered}
$$

Requiring $I^{\prime}=\left\{\sigma^{j}, \omega^{1}, \cdots, \omega^{i}\right\}$ is an extended closed ideal, we may derive the following equation from Equation (4)

$$
\frac{1}{2}\left(F_{r s}^{a} \lambda_{a}^{i} \mathrm{~d} x^{r} \wedge \mathrm{d} x^{s}+M_{l k}^{i} \omega^{k} \wedge \omega^{l}\right)=f_{\delta}^{i} \sigma^{\delta}+\eta_{l}^{i} \wedge \omega^{l},
$$

where $f_{\delta}^{i}$ and $\eta_{l}^{i}$ are the zero and one forms on the base manifold $X$, respectively. Equation (6) can decomposed into the following equations: 


$$
\frac{1}{2} F_{r s}^{a}(X) \lambda_{a}^{i}(y) \mathrm{d} x^{r} \wedge \mathrm{d} x^{s}=f_{\delta}^{i} \sigma^{\delta}, \quad \frac{1}{2} M_{l k}^{i}(Y) \omega^{k} \wedge \omega^{l}=\eta_{l}^{i} \wedge \omega^{l},
$$

Equation (7) is called the fundamental equation of the prolongation structure [26] [27]. In general, we may completely determine the prolongation structure of a given nonlinear system when the solution of the one fundamental equation can be found.

\section{Covariant Prolongation Structure of Gross-Pitaevskii Equation in the Bose-Einstein Condensate}

In order to express Equation (1) in differential forms, we add the conjugate equation of Equation (1) to Equation (1) and obtain the following system :

$$
\begin{gathered}
-i q_{t}^{*}+q_{x x}^{*}+2 \mu^{2}|q|^{2} q^{*}+\left(-i \beta-\alpha x+\beta^{2} x^{2}\right) q^{*}=0, \\
i q_{t}+q_{x x}+2 \mu^{2}|q|^{2} q+\left(i \beta-\alpha x+\beta^{2} x^{2}\right) q=0 .
\end{gathered}
$$

We define the independent variables as $X=\left\{x, t, q, q_{x}, q^{*}, q_{x}^{*}\right\}=\left\{x_{1}, x_{2}, x_{3}, x_{4}, x_{5}, x_{6}\right\}$. The Gross-Pitaevskii equation can then be expressed in the following set of two-forms given by [26] [27]

$$
\begin{aligned}
& \sigma^{1}=\mathrm{d} x_{3} \wedge \mathrm{d} x_{2}-x_{4} \mathrm{~d} x_{1} \wedge \mathrm{d} x_{2}, \\
& \sigma^{2}=\mathrm{d} x_{1} \wedge \mathrm{d} x_{3}-i \mathrm{~d} x_{4} \wedge \mathrm{d} x_{2}-i\left[2 \mu^{2} x_{3} x_{5}+\left(i \beta-\alpha x_{1}+\beta^{2} x_{1}^{2}\right)\right] x_{3} \mathrm{~d} x_{1} \wedge \mathrm{d} x_{2}, \\
& \sigma^{3}=\mathrm{d} x_{5} \wedge \mathrm{d} x_{2}-x_{6} \mathrm{~d} x_{1} \wedge \mathrm{d} x_{2}, \\
& \sigma^{4}=-\mathrm{d} x_{1} \wedge \mathrm{d} x_{5}-i \mathrm{~d} x_{6} \wedge \mathrm{d} x_{2}-i\left[2 \mu^{2} x_{3} x_{5}+\left(-i \beta-\alpha x_{1}+\beta^{2} x_{1}^{2}\right)\right] x_{5} \mathrm{~d} x_{1} \wedge \mathrm{d} x_{2},
\end{aligned}
$$

where the letter $d$ denotes the exterior derivative and the symbol $\wedge$ represents the exterior product. In order to ensure complete equivalence between the forms (9) and the Gross-Pitaevskii Equation (8), the ideal $I$ must be closed, i.e., $\mathrm{d} I \subset I$. In this closed ideal any local surface element which annuls the $\sigma^{j}$ also annuls their exterior derivatives $\mathrm{d} \sigma^{j}$. In order to establish the prolongation structure, we extend the above ideal by adding to it a connection 1-forms, defined by [26] [27]

$$
\omega^{l}=\mathrm{d} y^{l}+\Gamma_{r}^{a}(X) \lambda_{a}^{l}(y) \mathrm{d} x^{r},
$$

where $X=\left\{x_{1}, x_{2}, x_{3}, x_{4}, x_{5}, x_{6}\right\}$, and $y^{l}$ are the prolongation variable. For some suitably chosen prolongation variables and imposing the closed condition of the extended ideal $I^{\prime}=\left\{\sigma^{j}, \omega^{1}, \cdots, \omega^{l}\right\}$ under covariant exterior derivative, it leads to the covariant fundamental equations.

Substituting the above two forms $\left.\sigma^{j}\right|_{j=1, \cdots, 4}$ into the fundamental equation Equation (7), we have [26] [27]

$$
\begin{gathered}
F_{1,2}^{l}-x_{4} F_{2,3}^{l}-x_{6} F_{2,5}^{l}+i\left[2 \mu^{2} x_{3} x_{5}+\left(i \beta-\alpha x_{1}+\beta^{2} x_{1}^{2}\right)\right] x_{3} F_{1,3}^{l} \\
-i\left[2 \mu^{2} x_{3} x_{5}+\left(-i \beta-\alpha x_{1}+\beta^{2} x_{1}^{2}\right)\right] x_{5} F_{1,5}^{l}=0, \\
F_{2,4}^{l}-i F_{1,3}^{l}=0, \quad F_{2,6}^{l}+i F_{1,5}^{l}=0, \quad F_{1,4}^{l}=F_{1,6}^{l}=0, \quad F_{r, s}^{l}=0,(r, s=4,5,6) .
\end{gathered}
$$

Then, substituting the first equation of Equation (5) into Equation (11), we 
have the following over-determined difference equations

$$
\begin{aligned}
& \frac{\partial \Gamma_{1}^{1}}{\partial x_{4}}=0, \frac{\partial \Gamma_{1}^{2}}{\partial x_{4}}=0, \quad \frac{\partial \Gamma_{1}^{3}}{\partial x_{4}}=0, \quad \frac{\partial \Gamma_{1}^{1}}{\partial x_{6}}=0, \quad \frac{\partial \Gamma_{1}^{2}}{\partial x_{6}}=0, \quad \frac{\partial \Gamma_{1}^{3}}{\partial x_{6}}=0 \\
& \frac{\partial \Gamma_{2}^{1}}{\partial x_{4}}+i \frac{\partial \Gamma_{1}^{1}}{\partial x_{3}}=0, \quad \frac{\partial \Gamma_{2}^{2}}{\partial x_{4}}+i \frac{\partial \Gamma_{1}^{2}}{\partial x_{3}}=0, \quad \frac{\partial \Gamma_{2}^{3}}{\partial x_{4}}+i \frac{\partial \Gamma_{1}^{3}}{\partial x_{3}}=0 \\
& \frac{\partial \Gamma_{2}^{1}}{\partial x_{6}}-i \frac{\partial \Gamma_{1}^{1}}{\partial x_{5}}=0, \quad \frac{\partial \Gamma_{2}^{2}}{\partial x_{6}}-i \frac{\partial \Gamma_{1}^{2}}{\partial x_{5}}=0, \quad \frac{\partial \Gamma_{2}^{3}}{\partial x_{6}}-i \frac{\partial \Gamma_{1}^{3}}{\partial x_{5}}=0 \\
& \frac{\partial \Gamma_{1}^{1}}{\partial x_{2}}-\frac{\partial \Gamma_{2}^{1}}{\partial x_{1}}-x_{4} \frac{\partial \Gamma_{2}^{1}}{\partial x_{3}}-x_{6} \frac{\partial \Gamma_{2}^{1}}{\partial x_{5}}+2 \Gamma_{1}^{2} \Gamma_{2}^{3}-2 \Gamma_{3}^{1} \Gamma_{2}^{2} \\
&+i\left[2 \mu^{2} x_{3} x_{5}+\left(i \beta-\alpha x_{1}+\beta^{2} x_{1}^{2}\right)\right] x_{3} \frac{\partial \Gamma_{1}^{1}}{\partial x_{3}} \\
&-i\left[2 \mu^{2} x_{3} x_{5}+\left(-i \beta-\alpha x_{1}+\beta^{2} x_{1}^{2}\right)\right] x_{5} \frac{\partial \Gamma_{1}^{1}}{\partial x_{5}}=0 \\
& \frac{\partial \Gamma_{1}^{2}}{\partial x_{2}}-\frac{\partial \Gamma_{2}^{2}}{\partial x_{1}}-x_{4} \frac{\partial \Gamma_{2}^{2}}{\partial x_{3}}-x_{6} \frac{\partial \Gamma_{2}^{2}}{\partial x_{5}}+\Gamma_{1}^{1} \Gamma_{2}^{2}-\Gamma_{1}^{2} \Gamma_{2}^{1} \\
&+i\left[2 \mu^{2} x_{3} x_{5}+\left(i \beta-\alpha x_{1}+\beta^{2} x_{1}^{2}\right)\right] x_{3} \frac{\partial \Gamma_{1}^{2}}{\partial x_{3}} \\
&-i\left[2 \mu^{2} x_{3} x_{5}+\left(-i \beta-\alpha x_{1}+\beta^{2} x_{1}^{2}\right)\right] x_{5} \frac{\partial \Gamma_{1}^{2}}{\partial x_{5}}=0 \\
&+i\left[2 \mu^{2} x_{3} x_{5}+\left(-i \beta-\alpha x_{1}+\beta^{2} x_{1}^{2}\right)\right] x_{5} \frac{\partial \Gamma_{1}^{3}}{\partial x_{5}}=0 \\
&+\frac{\partial \Gamma_{1}^{3}}{\partial x_{2}}-\frac{\partial \Gamma_{2}^{3}}{\partial x_{1}}-x_{4} \frac{\partial \Gamma_{2}^{3}}{\partial x_{3}}-x_{6} \frac{\partial \Gamma_{2}^{3}}{\partial x_{5}}-\Gamma_{1}^{1} \Gamma_{2}^{3}+\Gamma_{1}^{3} \Gamma_{2}^{1} \\
&+i\left[2 \mu^{2} x_{3} x_{5}+\left(i \beta-\alpha x_{1}+\beta^{2} x_{1}^{2}\right)\right] x_{3} \frac{\partial \Gamma_{1}^{3}}{\partial x_{3}} \\
&+f_{1}
\end{aligned}
$$

Solving the over-determined difference equations Equation (12), we obtain the following solutions

$$
\begin{gathered}
\Gamma_{1}^{1}=\frac{-i \alpha}{2 \beta}-2 i \lambda \exp \left(-2 \beta x_{2}\right), \\
\Gamma_{1}^{2}=\mu x_{3} \exp \left(-i \beta x_{1}^{2} / 2\right), \\
\Gamma_{1}^{3}=-\mu x_{5} \exp \left(i \beta x_{1}^{2} / 2\right), \\
\Gamma_{2}^{1}=-4 i\left(\frac{\alpha}{4 \beta}+\lambda \exp \left(-2 \beta x_{2}\right)\right)^{2}+4 i \beta \lambda x_{1} \exp \left(-2 \beta x_{2}\right)+2 i \mu^{2} x_{3} x_{5}, \\
\Gamma_{2}^{2}=\left[\frac{\alpha \mu}{2 \beta} x_{3}+2 \mu \lambda x_{3} \exp \left(-2 \beta x_{2}\right)-\mu \beta x_{1} x_{3}+i \mu x_{4}\right] \exp \left(-i \beta x_{1}^{2} / 2\right), \\
\Gamma_{2}^{3}=\left[\frac{-\alpha \mu}{2 \beta} x_{5}-2 \mu \lambda x_{5} \exp \left(-2 \beta x_{2}\right)+\mu \beta x_{1} x_{5}+i \mu x_{6}\right] \exp \left(i \beta x_{1}^{2} / 2\right),
\end{gathered}
$$

with $\lambda$ as the hidden spectral parameter and the other components are zero.

Let us use the two dimensional linear representation of $\operatorname{SL}(2, \mathrm{R})$ [22] [23] 
given by,

$$
X_{1}=-\frac{1}{2} Y_{1} \frac{\partial}{\partial Y_{1}}+\frac{1}{2} Y_{2} \frac{\partial}{\partial Y_{2}}, \quad X_{2}=-Y_{2} \frac{\partial}{\partial Y_{1}}, \quad X_{3}=-Y_{1} \frac{\partial}{\partial Y_{2}} .
$$

Setting the transformation $X_{m}=\lambda_{m}^{i}(Y) \frac{\partial}{\partial Y_{i}}$, which leads to

$$
\lambda_{1}^{1}=-\frac{1}{2} Y_{1}, \quad \lambda_{1}^{2}=\frac{1}{2} Y_{2}, \quad \lambda_{2}^{1}=-Y_{2}, \quad \lambda_{2}^{2}=0, \quad \lambda_{3}^{1}=0, \quad \lambda_{3}^{2}=-Y_{1},
$$

we therefore derive the following Lax-Pairs, given by

$$
\begin{aligned}
& Y_{x}=-\left[\Gamma_{1}^{2} T_{-1}+\Gamma_{1}^{1} T_{0}+\Gamma_{1}^{3} T_{1}\right] Y, \\
& Y_{t}=-\left[\Gamma_{2}^{2} T_{-1}+\Gamma_{1}^{2} T_{0}+\Gamma_{2}^{3} T_{1}\right] Y,
\end{aligned}
$$

where $\left.T_{i}\right|_{i=-1, \cdots, 1}$ represent the generators of the SL(2,R)-symmetry [22] [23].

On the other hand, by selecting the matrix representation of a generators of a $\mathrm{SL}(2, \mathrm{C})$ symmetry, the Lax-representation associated to such an algebra is then given by

$$
\begin{gathered}
Y_{x}=\left[\Gamma_{1}^{2} e_{-}+\frac{\Gamma_{1}^{1}}{2} h+\Gamma_{1}^{3} e_{+}\right] Y, \\
Y_{t}=\left[\Gamma_{2}^{2} e_{-}+\frac{\Gamma_{2}^{1}}{2} h+\Gamma_{2}^{3} e_{+}\right] Y,
\end{gathered}
$$

where $\left(e_{ \pm}, h\right)$ are the generators of $\operatorname{SL}(2, \mathrm{C})$ Lie algebra [8].

Besides the previous symmetries, we select the generators of the $\mathrm{SU}(1,1)$ symmetry [23] and we obtain the following Lax-representation

$$
\begin{aligned}
& Y_{x}=\left[\Gamma_{1}^{1} T_{1}+\left(\Gamma_{1}^{2}+\Gamma_{1}^{3}\right) T_{2}+\left(\Gamma_{1}^{2}-\Gamma_{1}^{3}\right) T_{3}\right] Y, \\
& Y_{t}=\left[\Gamma_{2}^{1} T_{1}+\left(\Gamma_{2}^{2}+\Gamma_{2}^{3}\right) T_{2}+\left(\Gamma_{2}^{2}-\Gamma_{2}^{3}\right) T_{3}\right] Y,
\end{aligned}
$$

where $\left.T_{i}\right|_{i=1, \cdots, 3}$ are the generators of $\mathrm{SU}(1,1)$ Lie algebra [23].

Another Lax-representation can be derived in the form

$$
\begin{aligned}
& Y_{x}=\left[i\left(\Gamma_{1}^{2}+\Gamma_{1}^{3}\right) T_{1}-\left(\Gamma_{1}^{2}-\Gamma_{1}^{3}\right) T_{2}+\Gamma_{1}^{1} T_{3}\right] Y, \\
& Y_{t}=\left[i\left(\Gamma_{2}^{2}+\Gamma_{2}^{3}\right) T_{1}-\left(\Gamma_{2}^{2}-\Gamma_{2}^{3}\right) T_{2}+\Gamma_{2}^{1} T_{3}\right] Y,
\end{aligned}
$$

and

$$
\begin{gathered}
Y_{x}=\left(-\Gamma_{1}^{2} T^{-1}+\Gamma_{1}^{1} T^{0}+\Gamma_{1}^{3} T^{1}\right) Y, \\
Y_{t}=\left(-\Gamma_{2}^{2} T^{-1}+\Gamma_{1}^{1} T^{0}+\Gamma_{2}^{3} T^{1}\right) Y,
\end{gathered}
$$

where $\left.T_{i}\right|_{i=1, \cdots, 3}$ and $\left.T^{i}\right|_{i=-1, \cdots, 1}$ are the generators of a $\mathrm{SU}(2)$-symmetry [22] [23] and centreless Virasoro Lie algebra [9] [10].

From the previous discussion, it appears that the dynamics of the the GrossPitaevskii (GP) equation modeled by Equation (1), are basically governed by internal structural symmetries, including the Virasoro algebra, $\mathrm{SL}(2, \mathrm{C}), \mathrm{SU}(2)$, $\mathrm{SU}(1,1)$ and $\mathrm{SL}(2, \mathrm{R})$. Such symmetries have some physical implications. For 
example, the SU(1,1) -symmetries show that the system (1) possesses some conserved quantities that are rotationally and hyperbolically invariant, respectively. Thus, we have shown that Eqution (1) is Lax integrable by giving its corresponding Lax-Pair Equations (16)-(20).

\section{Conservation Laws of the Gross-Pitaevskii Equation in the Bose-Einstein Condensate}

In the following, we will prove the existence of infinitely-many conservation laws, which further verifies the integrability of Equation (1).

By means of the one dimensional linear representation of $\operatorname{SL}(2, R)$, we derive the Riccati equations

$$
\begin{gathered}
Y_{x}=\Gamma_{1}^{3}-\Gamma_{1}^{1} Y-\Gamma_{1}^{2} Y^{2} \\
Y_{t}=\Gamma_{2}^{3}-\Gamma_{2}^{1} Y-\Gamma_{2}^{2} Y^{2} .
\end{gathered}
$$

Then setting [40],

$$
\begin{gathered}
Y=\rho Y_{-1}+Y_{0}+\rho^{-1} Y_{1}+\rho^{-2} Y_{2}+\rho^{-3} Y_{3}+\rho^{-4} Y_{4}+\rho^{-5} Y_{5}+\cdots, \quad(\zeta=\rho) \\
Q=x_{3} \exp \left(-i \beta x_{1}^{2} / 2\right), \quad \zeta=\frac{\alpha}{4 \beta}+\lambda \exp \left(-2 \beta x_{2}\right),
\end{gathered}
$$

and substituting it into Equation (21), then comparing the coefficient of $\rho^{k}$, we have

$$
\begin{aligned}
& k=2: Y_{-1}\left(2 i-\mu Q Y_{-1}\right)=0, \\
& k=1: Y_{-1, x}=Y_{0}\left(2 i-2 \mu Q Y_{-1}\right), \\
& k=0: Y_{0, x}=-\mu Q^{\star}+Y_{1}\left(2 i-2 \mu Q Y_{-1}\right)-\mu Q Y_{0}^{2}, \\
& k=-1: Y_{1, x}=2 i Y_{2}-2 \mu Q\left(Y_{-1} Y_{2}+Y_{0} Y_{1}\right), \\
& k=-2: Y_{2, x}=2 i Y_{3}-\mu Q\left(Y_{1}^{2}+2 Y_{-1} Y_{3}+2 Y_{0} Y_{2}\right), \\
& \quad \vdots \\
& k=j: Y_{j, x}=\left(2 i-2 \mu Q Y_{-1}\right) Y_{j+1}-\mu Q\left(\sum_{m=0}^{j} Y_{m} Y_{j-m}\right),
\end{aligned}
$$

from which we obtain

$$
\begin{gathered}
Y_{-1}=\frac{2 i}{\mu Q}, \quad Y_{0}=\frac{1}{\mu} Q_{x} Q^{-2}, \quad Y_{1}=\frac{1}{2 i \mu}\left[\left(Q_{x} Q^{-2}\right)_{x}+\mu^{2}\left(Q^{\star}+Q_{x}^{2} Q^{-3}\right)\right], \\
Y_{j+1}=\frac{Y_{j, x}+\mu Q\left(\sum_{m=0}^{j} Y_{m} Y_{j-m}\right)}{\left(2 i-2 \mu Q Y_{-1}\right)} .
\end{gathered}
$$

From the compatibility condition, the infinitely-many conservation laws for Equation (1) can be expressed as [41]

$$
\frac{\partial D_{j}}{\partial x}=\frac{\partial F_{j}}{\partial t}, \quad j=-1, \cdots, \infty
$$

where the conserved density $D_{j}$ and the conserved flow $F_{j}$ are the following $D_{-1}=2 \mu Q Y_{0}+\left(i \mu Q_{x}-2 \mu \beta Q x\right) Y_{-1}, \quad D_{0}=2 \mu Q Y_{1}+\left(i \mu Q_{x}-2 \mu \beta Q x\right) Y_{0}+i \mu^{2} Q Q^{\star}$, $D_{1}=2 \mu Q Y_{2}+\left(i \mu Q_{x}-2 \mu \beta Q x\right) Y_{1}, \quad D_{j}=2 \mu Q Y_{j+1}+\left(i \mu Q_{x}-2 \mu \beta Q x\right) Y_{j}, \quad j=2, \cdots,+\infty$ 


$$
F_{j}=\mu Q Y_{j}, \quad j=-1, \cdots,+\infty .
$$

Using the vanishing boundary condition, we can give the three constants of motions from the obtained conservation laws,

$$
\int_{-\infty}^{+\infty} D_{-1} \mathrm{~d} t=\int_{-\infty}^{+\infty} D_{0} \mathrm{~d} t=\int_{-\infty}^{+\infty} D_{1} \mathrm{~d} t=\text { constant. }
$$

\section{Exact multisoliton solutions of the Gross-Pitaevskii Equation in the Bose-Einstein Condensate}

In order to derive the analytical soliton solutions to Equation (1), we will employ the Hirota bilinear method [42] [43] [44], which is an efficient and direct approach to construct soliton solutions to nonlinear evolution equations via the bilinear forms from the dependent variables transformation.

To get the bilinear forms for Equation (1) we introduce the dependent variable transformation

$$
q\left(x_{1}, x_{2}\right)=\frac{g\left(x_{1}, x_{2}\right)}{f\left(x_{1}, x_{2}\right)} \exp \left(i \beta x_{1}^{2} / 2\right)
$$

where $g\left(x_{1}, x_{2}\right)$ is the complex differentiable function, and $f\left(x_{1}, x_{2}\right)$ is a real one. Substituting relation (28) into Equation (1), the bilinear equations of Equation (1) turns out to be in the following forms

$$
\begin{gathered}
{\left[i \mathrm{D}_{x_{2}}+2 i \beta x_{1} \mathrm{D}_{x_{1}}+\left(2 i \beta-\alpha x_{1}\right)+\mathrm{D}_{x_{1}}^{2}\right] g \cdot f=0,} \\
\mathrm{D}_{x_{1}}^{2} f \cdot f=2 \mu^{2} g \cdot g^{\star},
\end{gathered}
$$

where D denotes the Hirota's derivative [42] [43] [44].

To construct the soliton solutions of Equation (1), we expand $g\left(x_{1}, x_{2}\right)$ and $f\left(x_{1}, x_{2}\right)$ with respect to a formal expansion parameter $\epsilon$ as

$$
\begin{gathered}
f=1+\epsilon^{2} f_{2}+\epsilon^{4} f_{4}+\cdots+\epsilon^{2 i} f_{2 i}+\cdots, \\
g=\epsilon g_{1}+\epsilon^{3} g_{3}+\cdots+\epsilon^{2 i+1} g_{2 i+1}+\cdots .
\end{gathered}
$$

where $g_{2 i+1}\left(x_{1}, x_{2}\right)$ is the complex differentiable function, and $f_{2 i}\left(x_{1}, x_{2}\right)$ is a real one

To derive the one-soliton solutions to Equation (1), we truncate expressions Equation (30) as $g=\epsilon g_{1}$ and $f=1+\epsilon^{2} f_{2}$, setting $\epsilon=1$ and substituting then into Bilinear forms Equation (29). We obtain the one-soliton solutions to Equation (1) as

$$
\begin{aligned}
q\left(x_{1}, x_{2}\right)= & \frac{A_{1}\left(\xi_{1}+\xi_{1}^{\star}\right)}{2 \mu\left|A_{1}\right|} \exp \left[\frac{1}{2}\left(\theta_{1}-\theta_{1}^{\star}\right)+\frac{i}{2} \beta x_{1}^{2}-2 \beta x_{2}\right] \\
& \times \operatorname{sech}\left[\frac{1}{2}\left(\theta_{1}+\theta_{1}^{\star}\right)+2 \beta x_{2}+2 \ln \left(\frac{\mu\left|A_{1}\right|}{\xi_{1}+\xi_{1}^{\star}}\right)\right]
\end{aligned}
$$

where

$$
\begin{aligned}
\theta_{j}\left(x_{1}, x_{2}\right)= & {\left[\frac{\alpha}{2 i \beta}+\xi_{j} \exp \left(-2 \beta x_{2}\right)\right] x_{1}+\left[\frac{\alpha^{2}}{4 i \beta^{2}}-2 \beta\right] x_{2} } \\
& +\frac{\xi_{j}^{2}}{4 i \beta} \exp \left(-4 \beta x_{2}\right)-\frac{\alpha \xi_{j}}{2 \beta} \exp \left(-2 \beta x_{2}\right)
\end{aligned}
$$




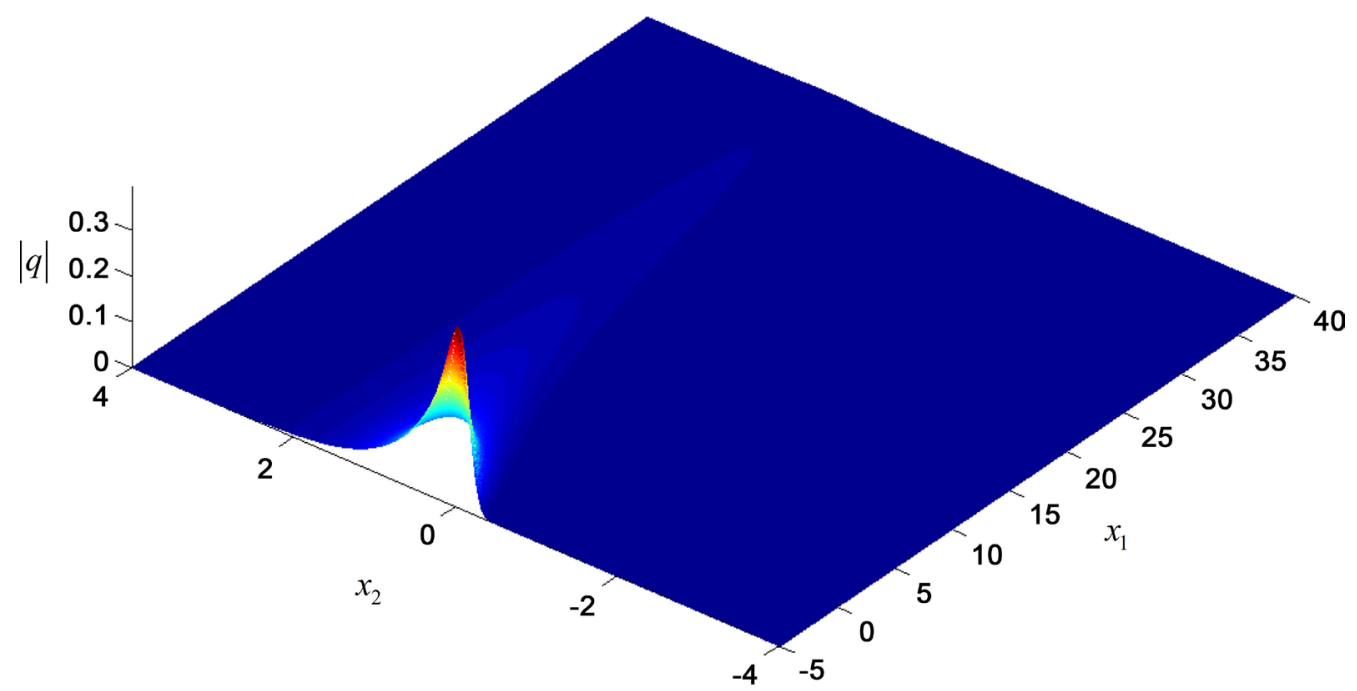

(a)

Figure 1. The one-soliton solution via expression (31) with parameters $\alpha=5 ; \beta=1 ; \xi_{1}=1+5 i$; $\mu=2,5 ; A_{1}=1$.

it is depicted in Figure 1.

Similarly, in order to derive the two-soliton solutions, we can choose

$$
\begin{gathered}
g_{1}=A_{1} \exp \left(\theta_{1}\right)+A_{2} \exp \left(\theta_{2}\right), \\
f_{2}=B_{1}^{1^{*}} \exp \left(\theta_{1}+\theta_{1}^{*}\right)+B_{1}^{2^{*}} \exp \left(\theta_{1}+\theta_{2}^{*}\right)+B_{2}^{1^{*}} \exp \left(\theta_{2}+\theta_{1}^{*}\right)+B_{2}^{2^{*}} \exp \left(\theta_{2}+\theta_{2}^{*}\right), \\
g_{3}=C_{11}^{1^{*}} \exp \left(2 \theta_{1}+\theta_{1}^{*}\right)+C_{11}^{2^{*}} \exp \left(2 \theta_{1}+\eta_{2}^{*}\right)+C_{12}^{1^{*}} \exp \left(\theta_{1}+\theta_{2}+\theta_{1}^{*}\right) \\
+C_{12}^{2^{*}} \exp \left(\theta_{1}+\theta_{2}+\theta_{2}^{*}\right)+C_{22}^{1^{*}} \exp \left(2 \theta_{2}+\theta_{1}^{*}\right)+C_{22}^{2^{*}} \exp \left(2 \theta_{2}+\theta_{2}^{*}\right), \\
f_{4}=D_{11}^{1^{*} 1^{*}} \exp \left(2 \theta_{1}+2 \theta_{1}^{*}\right)+D_{22}^{1^{*} 1^{*}} \exp \left(2 \theta_{2}+2 \theta_{1}^{*}\right)+D_{11}^{2^{*} 2^{*}} \exp \left(2 \theta_{1}+2 \theta_{2}^{*}\right) \\
+D_{22}^{2^{*} 2^{*}} \exp \left(2 \theta_{2}+2 \theta_{2}^{*}\right),
\end{gathered}
$$

where

$$
\begin{gathered}
B_{m}^{n^{*}}=\frac{\mu^{2} A_{m} A_{n}^{*}}{4\left(\theta_{m, x_{1}}+\theta_{n, x_{1}}^{*}\right)^{2}}, \quad C_{m m}^{p^{*}}=-\frac{M_{m m p}^{-}(\theta) A_{m} B_{m}^{p^{*}}}{M_{m m p}^{+}}, \quad\left(\begin{array}{l}
p=1, \cdots, 2 \\
m=n=1, \cdots, 2
\end{array}\right), \\
C_{m n}^{p^{*}}=-\frac{M_{m n p}^{-}(\theta) A_{m} B_{n}^{p^{*}}+M_{n p m}^{-}(\theta) A_{n} B_{p}^{m^{*}}}{M_{m n p}^{+}}, \quad\left(\begin{array}{l}
p=1, \cdots, 2 \\
m=1, \cdots n=2
\end{array}\right), \\
M_{m n p}^{\mp}(\theta)=\left[i\left(\theta_{m, x_{2}} \mp \theta_{n, x_{2}} \mp \theta_{p, x_{2}}^{*}\right)+\left(\theta_{m, x_{1}} \mp \theta_{n, x_{1}} \mp \theta_{p, x_{1}}^{*}\right)^{2}\right. \\
\left.+2 i \beta\left(\theta_{m, x_{1}} \mp \theta_{n, x_{1}} \mp \theta_{p, x_{1}}^{*}\right)+\left(2 i \beta-\alpha x_{1}\right)\right], \\
D_{m n}^{p l^{*}}=-\frac{C_{m n}^{p^{*}} C_{n}^{p l^{*}}}{B_{m}^{p^{*}}\left(\theta_{m, x}+\theta_{p, x}^{*}\right)^{2}}, \quad\left(\begin{array}{l}
p=l=1, \cdots, 2 \\
m=n=1, \cdots 2
\end{array}\right) .
\end{gathered}
$$

The two-soliton solutions to Equation (1) is written as

$$
q\left(x_{1}, x_{2}\right)=\frac{\left(g_{1}+g_{3}\right) \exp \left(\frac{i \beta x_{1}^{2}}{2}\right)}{1+f_{2}+f_{4}} .
$$




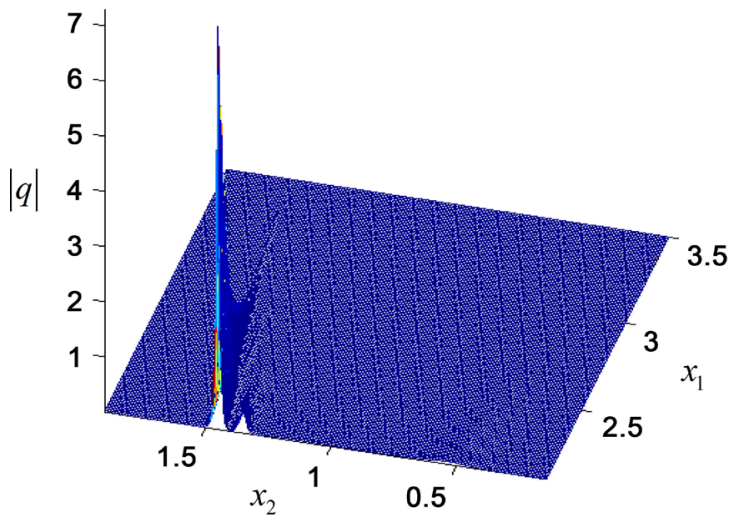

(a)

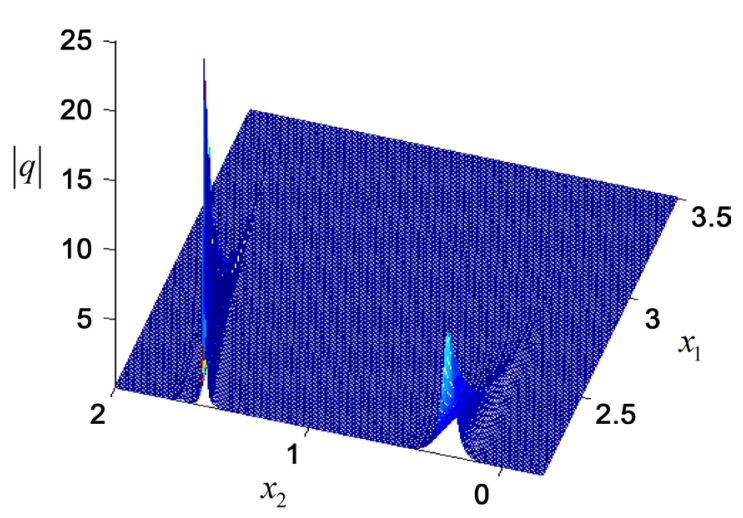

(b)

Figure 2. The two-soliton solutions via expression (35) with parameters (a) $\alpha=0.59 ; \beta=-0.98$; $\xi_{1}=-0.45-0.3 i ; \quad \xi_{2}=0.18+0.2 i ; \mu=1.5 ; \quad A_{1}=-1+i ; \quad A_{2}=1+i$, (b) $\alpha=0.59 ; \beta=-0.98 ; \quad \xi_{1}=-0.45-0.3 i$; $\xi_{2}=0.18+0.2 i ; \quad \mu=1.19 ; \quad A_{1}=-0.78+7.9 i ; \quad A_{2}=-0.65+0.2 i$.

and the corresponding depiction is shown in Figure 2.

If one and two-soliton solutions are calculated, then it is possible to generate the multi-soliton solution in the same way.

\section{Conclusion}

Throughout the present paper, we investigated the prolongation structure of the Gross-Pitaevskii equation which describes the propagation of an electron plasma wave packet with a large wavelength and small amplitude in a medium with a parabolic density and constant interactional damping from the viewpoint of covariant prolongation structure. As a result, we have unearthed some hidden structural symmetries governing the dynamics of the Gross-Pitaevskii equation such as $\mathrm{SL}(2, \mathrm{R}), \mathrm{SL}(2, \mathrm{C})$, Virasoro algebra, $\mathrm{SU}(1,1)$ and $\mathrm{SU}(2)$. Such symmetries have some physical implications. For example, the $\mathrm{SU}(1,1)$ -symmetries show that the system (1) possesses some conserved quantities that are rotationally and hyperbolically invariant, respectively. Thus, we have shown that Equation (1) is Lax integrable by giving its corresponding Lax-Pair Equations (16)-(20). In addition, infinite number of conservation Laws, one and two soliton solutions using Hirota bilinear method have been constructed. The prolongation structure analysis performed in the present study to the system (1) has revealed an infinite number of conserved quantities which stand as strong proof of integrability of this equation.

\section{Acknowledgements}

The authors would like to express their sincere thanks to the editors, anonymous reviewers and all the members of our discussion group for their critical comments and appropriate suggestions which have made this paper more precise and readable.

\section{References}

[1] Christiansen, P.L., Eilbeck, J.C. and Parmentier, R.D. (1993) Future Directions of 
Nonlinear Dynamics in Physical and Biological Systems. Plenum Press, New York, Vol. 312, 1-555.

[2] Sulem, C. and Sulem, P.L. (1999) The Nonlinear Schrodinger Equation Self-Focusing and Wave Collapse. Springer-Verlag, New York, Vol. 139, 1-347

[3] Hasegawa, A. and Matsumoto, M. (2003) Optical Solitons in Fiber. Springer-Verlag, Berlin, Heidelberg, New York. https://doi.org/10.1007/978-3-540-46064-0

[4] Gupta, M.R. (1979) Exact Inverse Scattering Solution of a Non-Linear Evolution Equation in a Non-Uniform Medium. Physics Letters A, 72, 420-422.

https://doi.org/10.1016/0375-9601(79)90833-8

[5] Uthayakumar, A., Han, Y.G and Lee, S.B. (2006) Soliton Solutions of Coupled Inhomogeneous Nonlinear Schrdinger Equation in Plasma. Chaos, Solitons \& Fractals, 29, 916-919. https://doi.org/10.1016/j.chaos.2005.08.055

[6] Wahlquist, H.D and Estabrook, F.B. (1975) Prolongation Structures of Nonlinear Evolution Equations. Journal of Mathematical Physics, 16, 1-7. https://doi.org/10.1063/1.522396

[7] Hermann, R. (1976) Pseudopotentials of Estabrook and Wahlquist, the Geometry of Solitons, and the Theory of Connections. Physical Review Letters, 36, 835-836. https://doi.org/10.1103/PhysRevLett.36.835

[8] Dodd, R. and Fordy, A. (1983) The Prolongation Structures of Quasi-Polynomial Flows. Proceedings of the Royal Society of London A, 385, 389-429. https://doi.org/10.1098/rspa.1983.0020

[9] Minoru, O. (1986) Prolongation Structures of Nonlinear Equations and Infinite-Dimensional Algebras. Journal of Mathematical Physics, 12, 2853-2860.

[10] Jinq-fa L. and Jing-ling, C. (1996) Lax Pair and Lie Algebraic Structures of Heterotic Liouville Systems by the Prolongation Approach. Physics Letters A, 213, 32-34. https://doi.org/10.1016/0375-9601(96)00100-4

[11] Zhai, Y., Albeverio, S., Zhao, Z.W. and Ke, W. (2006) Prolongation Structure of the $(2+1)$-Dimensional Integrable Heisenberg Ferromagnet Model. Journal of Physics $A, 39,2117-2126$. https://doi.org/10.1088/0305-4470/39/9/008

[12] Duan, X.J., Deng, M., Zhao, Z.W. and Ke, W. (2007) The Prolongation Structure of the Inhomogeneous Equation of the Reaction Diffusion Type. Journal of Physics $A$, 40, 3831-3837. https://doi.org/10.1088/1751-8113/40/14/006

[13] Tao, X. and Li, L.M. (2008) Covariant Prolongation Structure of Konno-Asai-Kakuhata Equation. Communications in Theoretical Physics, 50, 565-567. https://doi.org/10.1088/0253-6102/50/3/05

[14] Yan, Z., Yao, S., Zhang, C. and Gegenhasi (2016) The Fermionic Covariant Prolongation Structure of the Super Generalized Hirota Equation. Modern Physics Letters $B, 30$, Article ID: 1650251 .

[15] Bracken, P. (2010) A Geometric Interpretation of Prolongation by Means of Connections. Journal of Mathematical Physics, 51, Article ID: 113502.

[16] Bracken, P. (2010) Integrability and Prolongation Structure of a Generalized Korteweg-de Vries Equation. Journal of Mathematics and Statistics, 6, 125-130. https://doi.org/10.3844/jmssp.2010.125.130

[17] Cao, Y.H. and Wang, D.S. (2010) Prolongation Structures of a Generalized Coupled Korteweg-de Vries Equation and Miura Transformation. Communications in Nonlinear Science and Numerical Simulation, 15, 2344-2349.

[18] Wang, D.S. (2010) Integrability of the Coupled KdV Equations Derived from Two-Layer Fluids: Prolongation Structures and Miura Transformations. Nonlinear Analysis, 73, 270-281. 
[19] Wang, D.S. (2010) Complete Integrability and the Miura Transformation of a Coupled KdV Equation. Applied Mathematics Letters, 23, 665-669.

[20] Wang, D.S. (2010) Integrability of a Coupled KdV System: Painlevé Property, Lax Pair and Bäcklund Transformation. Applied Mathematics Letters, 216, 1349-1354.

[21] Ming, D. and Li, L.M (2010) Covariant Prolongation Structure of Coupled Inhomogeneous Nonlinear Schrödinger Equation. Communications in Theoretical Physics, 53, 218-222. https://doi.org/10.1088/0253-6102/53/2/03

[22] Thomas, B.B., Souleymanou, A., Kuetche, K.V., Mouna, F. and Kofane, T.C. (2011) Jet Bundle Formalism towards the Extension of the Stretched Rope Equation. Journal of Mathematical Analysis and Applications, 377, 269-273.

[23] Souleymanou, A., Thomas, B.B. and Kofane, T.C. (2013) Generating a New Higher-Dimensional Coupled Integrable Dispersionless System: Algebraic Structures, Bäcklund Transformation and Hidden Structural Symmetries. Communications in Theoretical Physics, 60, 145-149. https://doi.org/10.1088/0253-6102/60/2/01

[24] Morris, H. (1977) A Prolongation Structure for the AKNS System and Its Generalization. Journal of Mathematical Physics, 18, 533-536.

https://doi.org/10.1063/1.523298

[25] Lu, Q.K., Guo, H.Y. and Wu, K. (1983) A Formulation of Nonlinear Gauge Theory and Its Applications. Communications in Theoretical Physics, 2, 1029-1038. https://doi.org/10.1088/0253-6102/2/2/1029

[26] Guo, H., Hsiang, Y. and Wu, K. (1982) Connection Theory of Fibre Bundle and Prolongation Structures of Nonlinear Evolution Equations. Communications in Theoretical Physics, 1, 495-505. https://doi.org/10.1088/0253-6102/1/4/495

[27] Wu, K., Guo, H.Y. and Wang, S.K. (1983) Prolongation Structures of Nonlinear Systems in Higher Dimensions. Communications in Theoretical Physics, 2, 1425 1437. https://doi.org/10.1088/0253-6102/2/5/1425

[28] Davis, K.B., Mewes, M.O., Andrews, M.R., van Druten, N.J., Durfee, D.S., Kurn, D.M. and Ketterle, W. (1995) Bose-Einstein Condensation in a Gas of Sodium Atoms. Physical Review Letters, 75, 3969-3973. https://doi.org/10.1103/PhysRevLett.75.3969

[29] Bradley, C.C., Sackett, C.A., Tollett, J.J. and Hulet, R.G. (1995) Evidence of Bose-Einstein Condensation in an Atomic Gas with Attractive Interactions. Physical Review Letters, 75, 1687-1690. https://doi.org/10.1103/PhysRevLett.75.1687

[30] Liang, Z.X., Zhang, Z.D. and Liu, W.M. (2005) Dynamics of a Bright Soliton in Bose Einstein Condensates with Time Dependent Atomic Scattering Length in an Expulsive Parabolic Potential. Physical Review Letters, 94, Article ID: 050402.

[31] Li, Q.Y., Li, Z.D., Li, L. and Fu, G.S. (2010) Nonautonomous Bright and Dark Solitons of Bose Einstein Condensates with Feshbach Managed Time Dependent Scattering Length. Optics Communications, 283, 3361-3366.

[32] Modugno, G., Ferrari, G., Roati, G., Brecha, R.J., Simoni, A. and Inguscio, M. (2001) Bose-Einstein Condensation of Potassium Atoms by Sympathetic Cooling. Science, 294, 1320-1322. https://doi.org/10.1126/science.1066687

[33] Fried, D.G., Killian, T.C., Willmann, L., Landhuis, D., Moss, S.C., Kleppner, D. and Greytak, T.J. (1998) Bose-Einstein Condensation of Atomic Hydrogen. Physical Review Letters, 81, 3811-3814. https://doi.org/10.1103/PhysRevLett.81.3811

[34] Yang, Z.Y., Zhao, L.C., Zhang, T., Feng, X.Q. and Xue, R.H. (2011) Dynamics of a Nonautonomous Soliton in a Generalized Nonlinear Schrödinger Equation. Physical Review E, 83, Article ID: 066602.

[35] Wang, H. and Li, B. (2011) Solitons for a Generalized Variable-Coefficient Nonli- 
near Schrödinger Equation. Chinese Physics B, 20, Article ID: 040203.

[36] Li, L., Li, Z.H., Li, S.Q. and Zhou, G.S. (2004) Modulation Instability and Solitons on a cw Background in Inhomogeneous Optical Fiber Media. Optics Communications, 234, 169-176.

[37] Gupta, M.R., Som, B.K. and Dasgupta, B. (1978) Exact Solution of Damped Nonlinear Schrödinger Equation for a Parabolic Density Profile. Physics Letters A, 69, 172-174.

[38] Ablowitz, M.J., Kaup, D.J., Newell, A.C. and Segur, H. (1973) Nonlinear-Evolution Equations of Physical Significance. Physical Review Letters, 31, 125-127. https://doi.org/10.1103/PhysRevLett.31.125

[39] Wright, O.C. and Forest, M.G. (2000) On the Bäcklund-Gauge Transformation and Homoclinic Orbits of a Coupled Nonlinear Schrödinger System. Physica D: Nonlinear Phenomena, 141, 104-116.

[40] Yang, Y.-Q. and Chen, Y. (2011) Prolongation Structure of the Equation Studied by Qiao. Communications in Theoretical Physics, 56, 463-466.

https://doi.org/10.1088/0253-6102/56/3/13

[41] Zhao, L.C., Yang, Z.Y., Ling, L.M. and Liu, J. (2011) Precisely Controllable Bright Nonautonomous Solitons in Bose Einstein Condensate. Physics Letters A, 375, 1839-1842.

[42] Hirota, R. (2004) The Direct Method in Soliton Theory. Cambridge University Press, Cambridge, 198. https://doi.org/10.1017/CBO9780511543043

[43] Hirota, R. and Ohta, Y. (1991) Hierarchies of Coupled Soliton Equations. I. Journal of the Physical Society of Japan, 60, 798-809. https://doi.org/10.1143/JPSJ.60.798

[44] Ma, W.X. and You, Y.C. (2005) Solving the Korteweg-de Vries Equation by Its Bilinear Form: Wronskian Solutions. Transactions of the American Mathematical Society, 357, 1753-1778. https://doi.org/10.1090/S0002-9947-04-03726-2

\section{Submit or recommend next manuscript to SCIRP and we will provide best service for you:}

Accepting pre-submission inquiries through Email, Facebook, LinkedIn, Twitter, etc. A wide selection of journals (inclusive of 9 subjects, more than 200 journals) Providing 24-hour high-quality service User-friendly online submission system Fair and swift peer-review system Efficient typesetting and proofreading procedure Display of the result of downloads and visits, as well as the number of cited articles Maximum dissemination of your research work

Submit your manuscript at: http://papersubmission.scirp.org/ Or contact jamp@scirp.org 